\title{
Analysis of English Spatial Preposition by Autonomy-dependence Alignment Model
}

\author{
Nan $\mathrm{Li}^{1,2}$, Miaomiao Zhu' \\ ${ }^{1}$ Communication University of China, Beijing, China. \\ ${ }^{2}$ Xingtai University, Xingtai, Hebei, China
}

Keywords: spatial preposition; autonomous component; dependent component; cognitive

\begin{abstract}
Autonomy-dependence alignment (ADA) model can analyze the valence relation among the composites of language. The alignment of autonomy and dependence is achieved by elaboration site and profile determinacy. Autonomy and dependence are aligned as composite structure. The ADA model, which is applied in the analysis of English spatial preposition, is plausible and of explanatory power.
\end{abstract}

\section{English Spatial Preposition}

The study of English spatial preposition focuses on cognitive analysis, its acquisition and its contrast with Chinese.

\subsection{Cognitive Analysis of English Spatial Preposition.}

Cognitive study of English spatial preposition is often analyzed from categorization theory, schema theory and prototype theory. The study includes the meaning, acquisition and metaphor of English spatial preposition. According to categorization theory, English spatial preposition is studied in different dimensional space to set its typicality and marginality. The core part of category is profile, and the demarcation line is not always clear (Dirven\& Verspoor, 1998). The marginal part of the category is atypical and non-core and belongs to the gray area (Ma Shuhong, 2008).

English spatial preposition is often used in metaphor to express emotion, time, quantity, and so on. "Up" expresses positive emotion, early in time, the increase in quantity, and "down" expresses negative emotion, late in time and the decrease in quantity, and so on. For example,

It is important to speed up the "launch" of Commonwealth human rights protection mechanisms.

Eyewitnesses said it was going down the (road) and just (went) right off the road into the trees.

The origin of the word can be dated up to last century.

I have a vase handed down from my great-grandmother.

\subsection{The Acquisition of English Spatial Preposition.}

From categorization theory, Xu Qingli, Liu Zhenqian and Cai Jinting(2014) studies the impact of landmark categorization to Chinese learners by analyzing an English spatial preposition in, which is also influenced by cognitive factor and English level of the learners. The author made a comparison of construing spatial preposition between English and Chinese, and found that different landmark causes different construal and different learning effects. The conclusion is that similarity has positive transfer and difference has negative transfer, which enlightens the teaching and learning of English spatial preposition. From spatial categorization, Ma Shuhong studied the typical factor, the spatial concept of mother tongue, the difference of semantic classification and language structure between English and Chinese, and their impact on the learning of English spatial preposition. The author made a list analysis of the differences between English and Chinese spatial prepositions and selects the research subjects to carry out the experiment. Finally, it is concluded that the Chinese learners should master the spatial cognitive rules of English when they learn English spatial prepositions, and also overcome the influence and interference of the spatial concept rules of their own Chinese. In this process, positive transfer and negative transfer will affect. 


\subsection{The Contrast of Spatial Preposition between English and Chinese.}

The cognitive construal and acquisition of English spatial prepositions are related to contrastive studies in English and Chinese. Scholars made contrastive analysis of English and Chinese spatial prepositions from the perspective of cognitive schema, cognitive domain, categorization and prototype theory, and help the learners to recognize the differences in spatial cognition between English and Chinese. The conclusion is that the cognitive schema is different in English and Chinese, and the effect of the acquisition is different. The more the cognitive schema differences between English and Chinese, the worse the effect of the acquisition. The above studies analyze English and Chinese spatial prepositions from different aspects. This paper studies it from the perspective of cognitive grammar, mainly autonomy-dependence alignment (ADA) model.

\section{Autonomy-dependence Alignment (ADA) Model}

\subsection{Autonomy-dependence Relationship.}

Autonomy and dependence are a pair of categories in philosophy, and they are asymmetrical relations between two things. Autonomous components are relatively independent and can exist independently, while dependent components are relatively dependent and rely on the autonomous components that are compatible with them. Autonomy-dependence relation is not absolute, but a degree problem, which can be interchangeable under certain circumstances. Autonomous components are usually entity nouns, have directivity, and do not need to be attached to other components. Dependent components usually refer to prepositions, adjectives, adverbs, verbs, etc. The spatial prepositions need to be attached to an entity noun to express meaning. For example, in the phrase on the book, on, a spatial preposition, means above... But it needs to work with the entity noun. Without the book, on loses its support.

Cognitive grammar defines autonomy-dependence relationship as follows: structure $\mathrm{D}$ is dependent on structure $\mathrm{A}$, if $\mathrm{A}$ is an explanation of a substructure highlighted in $\mathrm{D}$. In other words, the meaning of $\mathrm{D}$ is dependent on the autonomous component A. For example, in the phrase on the desk, the substructure of [ON] corresponding to [THE-DESK] profile is the main landmark of [ON], and it is included in the profile of [ON], so the salience is very high. The meaning of on presupposes the existence of the desk, and the relationship between the desk and on is an autonomy-dependence relationship. Niu Baoyi (2008) believes that the elaboration of semantic salience of the autonomous components to the substructure of the dependent components is actually a concrete example of the former. The autonomy-dependence relationship between on and the desk can be considered as a categorization relationship, and the substructure of the desk (the entity consisting of table legs and table surfaces) is categorized as the subcategory of the substructure of on.

According to Langacker, autonomy-dependence relationship is relative. If $\mathrm{X}$ and $\mathrm{Y}$ are autonomy-dependence relationship, $\mathrm{Dx} \rightarrow \mathrm{y}$ indicates that $\mathrm{X}$ depends on $\mathrm{Y}$, dependence is only a degree problem, that is, to some extent, it may also be $\mathrm{Dy} \rightarrow \mathrm{x}$, indicating that $\mathrm{Y}$ is also dependent on $\mathrm{X}$. So that the comparison between $\mathrm{Dx} \rightarrow \mathrm{y}$ and $\mathrm{Dy} \rightarrow \mathrm{x}$ is important. If $\mathrm{Dx} \rightarrow \mathrm{y}$ is larger than $\mathrm{Dy} \rightarrow \mathrm{x}$, then $\mathrm{X}$ is identified as dependent structures, and $\mathrm{Y}$ as autonomous components, vice versa. For the phrase on the desk, if on is the dependent component, then its substructure landmark is prominent. With landmark, which can be explained in detail by the autonomous component the desk, on can be elaborated better. If in turn, the desk is regarded as the dependent component and is dependent on on, the substructure landmark highlighted by on is abstract, and can not explain the entity of the desk accurately. It can be seen that in this case, the dependency degree of the desk on on is weaker than the dependency degree of on on the desk, so we usually regard the desk as autonomous component and regard on as dependent component.

\subsection{Autonomy-dependence Alignment (ADA) Model.}

The ADA model is the framework of the valence relationship in the analysis language proposed by Langacker, which can clearly indicate the elaboration and correspondence between the substructures 
of the dependent components and the semantic profile of the autonomous components and the composite of the two. The semantic expression of the composite structure is illustrated by the relationship between the semantic salience and the semantic profile of autonomous components and dependent components. The model is as following:

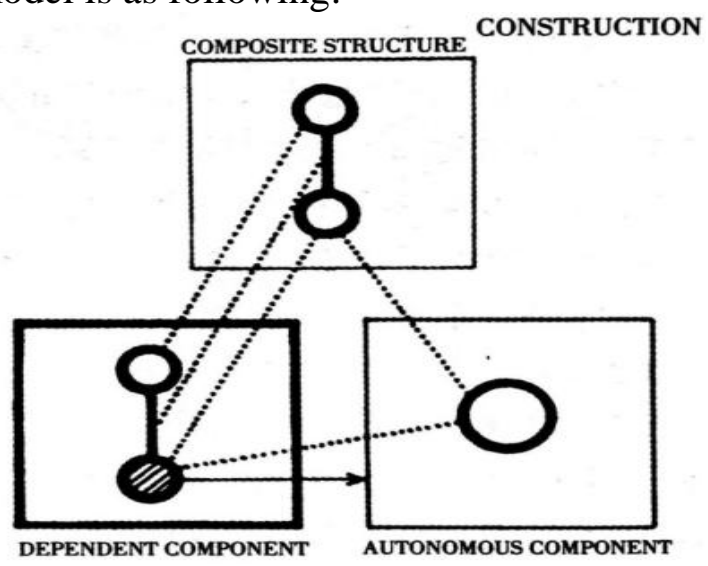

Fig.1

The lower two boxes in the diagram are dependent component (left) and autonomous component (right), and the upper box is the composite structure of the two. The small white circle in the dependent component represents the schematic substructure highlighted by the dependent components, and the shaded small circle represents the elaboration site in the dependency description; the rough real line between the two circles represents the relationship between the two; the arrow represents the elaboration relationship; the small circle in the autonomous component represents the profile or substructure of the autonomous component. In the upper box, the upper circle corresponds to the substructure highlighted in the dependent component, and the lower circle corresponds to the elaboration site of the dependent component and the substructure highlighted by the autonomous component.

\section{The Application of ADA Model into English Spatial Preposition}

As mentioned above, ADA model is used to analyze the autonomy-dependence relationship between the components of a grammatical structure. Using this model to explain English Spatial Prepositions enables us to understand them in a clear way. The following is a case study of under, around and between to verify the explanatory power of ADA model on English spatial prepositions.

Under The Boat.

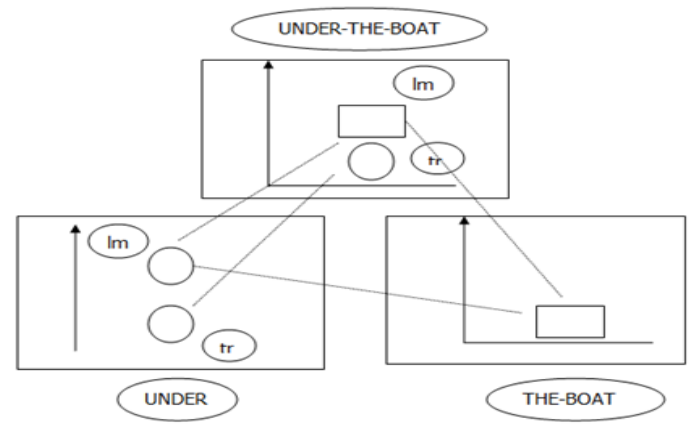

Fig.2

This diagram represents the autonomy-dependency relationship of the autonomic component [THE-BOAT] and the dependence component [UNDER] and its composite structure [UNDER-THE-BOAT]. The lower left diagram shows the relationship between trajector and landmark of under; the lower right diagram represents the entity of the boat; the upper figure is the composite structure of the two. In the under diagram, an abstract substructure landmark is highlighted, which is linked by the virtual line with boat. The concrete meaning of boat illustrates the substructure 
landmark; after the two is synthesized as under the boat, the specific trajector and the landmark are respectively corresponding to the dotted lines, showing the meaning of under the boat clearly.

Around The Fence.

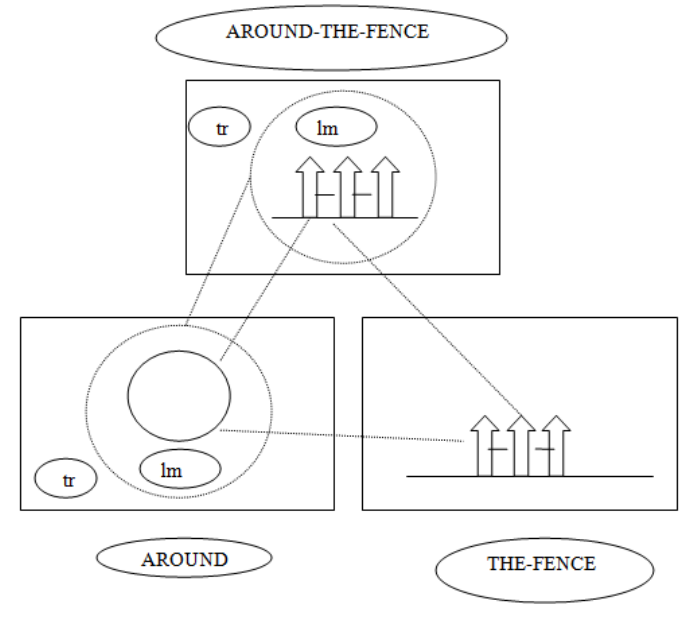

Fig.3

This diagram represents the autonomy-dependence relations of the autonomic component [THE-FENCE] and the dependent component [AROUND] and its composite structure [AROUND -THE- FENCE]. The lower left diagram shows the relationship between trajector and landmark of around; the lower right diagram represents the entity of the fence; the upper figure is the composite structure of the two. There are two circles in the around diagram, the outer ring is the trajector with the landmark as the reference material, which means the surround. The inner circle is a real line and highlights an abstract substructure landmark, which is aligned to the fence by the dotted line. The concrete meaning of the right lower diagram illustrates the substructure landmark of around; the two is synthesized into around the fence. In the composite structure, the fence in the circle is a landmark, not only corresponding to the landmark in the left lower figure, but also with the fence in the right lower figure, and the outer dashed circle means around, which clearly show the meaning of the in the box.

Between The Door and The Window.

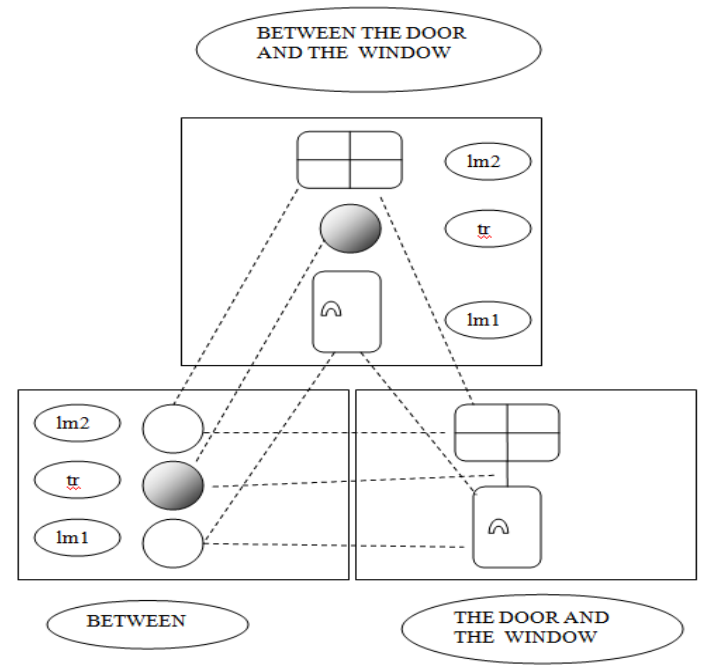

Fig.4

The diagram is slightly complex, representing the autonomy-dependence relationship of the autonomous component [THE-DOOR-AND-THE- WINDOW] and the dependent component [BETWEEN] and their composite structure [BETWEEN -THE- DOOR-AND-THE-WINDOW]. The left lower part is dependent component, the two white circles represent two landmarks, and a shadow circle in the middle represents the trajector between two landmarks. The right lower diagram is an 
autonomous component, corresponding to the two landmarks in the left lower diagram with the dotted line, and the entity in the right lower diagram illustrates the two abstract substructure landmarks in the left lower diagram. The upper diagram is the composite structure of the lower two. The shaded circle represents the components between the two boundaries, and the two landmarks correspond to the autonomous components and the dependent components respectively. The overall diagram clearly shows the meaning of between the door and the window.

\section{Cognitive Feature of English Spatial Preposition under ADA Model}

The ADA model can be applied to the analysis of English spatial prepositions. Spatial prepositions are usually dependent components, and their concrete meaning depends on a noun phrase. The alignment between English spatial prepositions and their attachment is based on autonomy-dependence relationship. They are aligned by hierarchy. The meaning of the composite structure is determined by the elaboration and correspondence between the internal structures of the autonomous components and dependent components. As mentioned earlier, asymmetry is the basic principle of an autonomous component and a dependent component in a valence relationship. In a valence relationship with an English spatial preposition, the preposition is usually a dependent component. The noun phrase, which is aligned to the preposition to get a larger structure, is usually the autonomous components, because the former is more dependent on the latter than the latter on the former.

\section{Conclusion}

This paper mainly analyzes the autonomy-dependence relationship, autonomy-dependence alignment, ADA model and its application in English spatial prepositions, which can enable people to understand English spatial prepositions from cognitive grammar. However, the examples of spatial prepositions in this paper are the structure of preposition + entity nouns, and noun phrases are substantive, which can make people understand their representation and profile clearly. In the actual situation, there are many combinations of spatial prepositions + abstract nouns / noun phrases, such as under attack, under repair. This article does not study such cases, therefore, there are deficiencies in the analysis, which need to be complemented one by one in the future.

\section{References}

[1] Dirven, R. \&M. Verspoor. Cognitive Exploration of Language and Linguistics, Philadelphia: John Benjamins Publishing Company, 1998.

[2] Langacker, R.W. Foundations of Cognitive Grammar, Vol.1. Theoretical Prerequisites. Stanford: Stanford University Press, 1987a.

[3] Ma Shuhong. The classification and acquisition of the semantic members of English spatial prepositions, Journal of PLA University of Foreign Languages, pp. 64-69, 2010.

[4] Niu Baoyi. Autonomy/Dependence Alignment (ADA)-an analysis model of cognitive grammar, Foreign Languages and Their Teaching, pp. 1-5, 2008.

[5] Xu Qingli, Liu Zhenqian and Cai Jinting. The influence of referential categorization on the use of English preposition in in Chinese Learners, Foreign Languages and Teaching Research, pp. 723-734, 2014. 